\title{
Kif4 regulates the expression of VEGFR1 through the PI3K/Akt signaling pathway in RAW264.7 monocytes/macrophages
}

\author{
YAN XU $^{1-3^{*}}$, YIJUN LUAN $^{1 *}$, SHAOHUA LIU $^{1}$, JINTANG SUN $^{2}$, KETAO WANG $^{1}$, \\ $\mathrm{JUN} \mathrm{CAI}^{3}$, WEN JIANG ${ }^{4}$, PISHAN YANG ${ }^{5}$, FENGCAI WEI $^{1}$ and XUN QU ${ }^{1,2}$ \\ ${ }^{1}$ Department of Stomatology and Institute of Stomatology, Qilu Hospital, Shandong University; \\ ${ }^{2}$ Institute of Basic Medical Sciences and Key Laboratory of Cardiovascular Proteomics, Qilu Hospital, \\ Shandong University; ${ }^{3}$ Jinan Stomalogic Hospital, Jinan, Shandong 250012, P.R. China; \\ ${ }^{4}$ Department of Biomedical Science, The University of Sheffield, Sheffield S10 2TN, UK; \\ ${ }^{5}$ School of Stomatology, Shandong University, Jinan, Shandong 250012, P.R. China
}

Received April 17, 2016; Accepted March 20, 2017

DOI: $10.3892 / \mathrm{ijmm} .2017 .2936$

\begin{abstract}
Kinesin superfamily protein 4 (Kif4), a microtubule-based motor protein, has been shown to participate in a number of critical cellular processes, such as cell division, the intracellular transport of membranous vesicles and signal transduction. However, whether KIF4 regulates vascular endothelial growth factor (VEGF) receptor 1 (VEGFR1) expression remains unknown. Thus, in this study, in order to examine the effects of Kif4 on the expression of VEGFR1 in RAW264.7 monocytes/macrophages, Kif4 was silenced using siRNA. RT-qPCR, western blot analysis and ELISA were used to assess the expression of Kif4 and VEGFR1 up- and downstream signaling molecules, including VEGF-A, VEGFR1, soluble form of VEGFR1 (sVEGFR1), phosphorylated (p-)Akt and Akt. The silencing Kif4 inhibited the mRNA expression of VEGF $(\mathrm{P}<0.01)$ and $\mathrm{p}-\mathrm{Akt}(\mathrm{P}<0.05)$; however, the level of VEGF-A was increased $(\mathrm{P}<0.05)$ compared with the negative control siRNA-transfected group. The silencing of Kif4 decreased the VEGFR1 mRNA $(\mathrm{P}<0.05)$, VEGFR1 protein and sVEGFR1 levels in the cell supernatant $(\mathrm{P}<0.01)$. Following the application of insulin-like growth factor-1 $(100 \mathrm{ng} / \mathrm{ml})$, the specific agonist of PI3K/Akt in the Kif4 siRNA-transfected
\end{abstract}

Correspondence to: Professor Xun Qu, Institute of Basic Medical Sciences and Key Laboratory of Cardiovascular Proteomics, Qilu Hospital, Shandong University, 107 Wenhuaxi Road, Jinan, Shandong 250012, P.R. China

E-mail: quxun@sdu.edu.cn

Professor Pishan Yang, School of Stomatology, Shandong University, 44-1 Wenhuaxi Road, Jinan, Shandong 250012, P.R. China

E-mail: yangps@sdu.edu.cn

*Contributed equally

Key words: kinesin superfamily protein 4, vascular endothelial growth factor receptor 1, vascular endothelial growth factor, Akt, monocytes/ macrophages group, the VEGFR1 mRNA levels $(\mathrm{P}<0.001)$, the VEGFR1 protein levels and the sVEGFR1 $(\mathrm{P}<0.01)$ levels significantly increased; however, the levels of VEGF in the cell supernatant were decreased $(\mathrm{P}<0.05)$. Taken together, these findings suggest that Kif4 regulates the expression of VEGFR1 in RAW264.7 cells and that the PI3K/Akt pathway is involved in this process.

\section{Introduction}

The kinesin superfamily proteins (Kifs) are microtubuledependent molecular motors and contain a conserved motor catalytic domain that binds to and hydrolyzes adenosine triphosphate (ATP) to produce chemical and mechanical energy. Kinesins play important roles in intracellular transport, mitosis, cellular morphogenesis and cellular functions. Kinesins not only transport various cargos, such as membranous organelles, protein complexes and mRNAs for the maintenance of basic cellular activity, but also play significant roles in brain growth, memory and the activity of neurons. It can be said that kinesins form the basis to living systems (1).

Kif4, a Kif member classified in kinesin-4 (2), is strongly expressed in juvenile tissues, including differentiated young neurons and is decreased considerably in adult mice, apart from the spleen (3). Kif4 has been reported as an essential factor involved in multiple cellular process, such as cell proliferation, DNA damage response, viral protein intracellular trafficking, immune cell activation and neuronal survival in brain development (4-9). It has also been suggested that Kif4 may be a primary initiating trigger for tumorigenesis (10).

Angiogenesis is a pathological process which is related to a wide range of diseases, from atherosclerosis to cancer (11). Central to the physiological regulation of angiogenesis is vascular endothelial growth factor (VEGF)-A and its receptors, involving VEGF receptor 1 (VEGFR1) and 2 (VEGFR2). VEGF-A displays potent angiogenic and vascular permeability activity. VEGFR2 is the crucial receptor for the functions of vascular endothelial cells. VEGFR1 is a high-affinity tyrosine kinase receptor for VEGF-A, a negative regulator of VEGFR2 
signaling capacity (12). VEGFR1 can be also generated as a short soluble form (sVEGFR1), consisting of only the extracellular ligand binding domain. Due to the lack of the transmembrane region, sVEGFR1 has the potential to act as a decoy receptor for VEGF-A by inhibiting the activation of VEGFR1 or VEGFR2 by binding to VEGF-A (13). Therefore, it may indirectly inhibit the pro-angiogenic activities VEGF-A.

It is known that sVEGFR1 is involved in the pregnancy disorder of pre-eclampsia, which is characterized by endothelial dysfunction (14) and the therapeutic potential of sVEGFR1 as an anti-angiogenic agent is gaining increasing attention in pre-clinical models of cancer. Thus, sVEGFR1 is a diagnostic marker and therapeutic target in angiogenesis-dependent diseases (15). In addition, VEGFR1-dependent disease reactions are also regulated by a balance of expression between the full-length and soluble forms of VEGFR1 (11).

Monocytes/macrophages represent immune effector cells, equipped with chemokine receptors and pathogen recognition receptors that mediate the migration from blood to tissues during infection (16). VEGFR1, which is well-expressed in monocytes/macrophages, is an important cell surface marker, as well as a biologically functional molecule for monocyte/macrophage lineages $(17,18)$. Through the receptor VEGFR1, monocytes/macrophages stimulate vascular biologyical responses in various tissues and promote a variety of diseases, such as tumor growth via pro-angiogenesis, tumor metastasis, lymphangiogenesis, arthritis, atherosclerosis and wound healing (18-26). Therefore, it is important to examine the expression of VEGFR1 in monocytes/macrophages and the underlying mechanisms.

In this study, we investigated whether Kif4 regulates VEGFR1, sVEGFR1 and VEGF-A expression in RAW264.7 monocytes/macrophages, and explored the underlying signaling mechanisms. Furthermore, we aimed to elucidate the regulatory mechanisms of VEGF-A and VEGFR1.

\section{Materials and methods}

Cell line and cell culture. The murine monocyte/macrophage cell line, RAW264.7, was obtained from the American Type Culture Collection (ATCC, Manassas, VA, USA) and routinely cultured in Dulbecco's modified Eagle's medium (DMEM) supplemented with $10 \%(\mathrm{v} / \mathrm{v})$ heat-inactivated fetal bovine serum (FBS) (both from Gibco-BRL, Carlsbad, CA, USA) at $37^{\circ} \mathrm{C}$ in a humidified air atmosphere and $5 \% \mathrm{CO}_{2}$. The RAW264.7 cells were plated at $5 \times 10^{5}$ cells/well into 6 -well plates and then treated with $1.5 \mathrm{ml}$ of medium.

Silencing of mouse Kif4 gene using small interfering RNA (siRNA). A siRNA expression vector was constructed by introducing synthetic double-stranded oligonucleotides as follows: Kif4 sense, 5'-GCUGAAGUUUAGGCAAUTT-3' and antisense, 5'-AUUGCCUAAACUUCUCAGCTT-3'; negative control (NC) sense, 5'-UUC UCCGAACGUGUCACGUTT-3' and antisense, 5'-ACGUGACACGUUCGGAGAATT-3' synthesized by GeneChem, Co., Ltd. (Shanghai, China) and green fluorescent FAM-labeled negative control siRNA (Shanghai GenePharma Co., Ltd., Shanghai, China) was used to detect the transfection efficiency. The RAW264.7 cells at $70 \%$ confluency were transfected with the Kif4 siRNA (si-Kif4) or NC siRNA (si-NC) or FAM labeled NC siRNA $(6 \mu 1)$ premixed with Lipofectamine 2000 (Invitrogen Life Technologies, Carlsbad, CA, USA) $(4.5 \mu \mathrm{l})$ in Opti-MEM in 6-well plates. The mock-transfected cells were transfected only with Lipofectamine ${ }^{\mathrm{TM}} 2000$ and were included as a control. After $6 \mathrm{~h}$, the cells were placed in fresh complete medium. In order to confirm whether siRNA was able to transfect into cells, the cells transfected with FAM labeled NC siRNA were observed under an inverted fluorescence microscope (serial no. 321463; Leica, Wetzlar, Germany). Cells were observed and photographed at x100 magnification under light field and fluorescence $(520 \mathrm{~nm})$, respectively. The transfection efficiency was determined by the positive rate of FAM green fluorescence from 5 different fields. To examine the effects of si-Kif4 on macrophage morphology, RAW 264.7 cells were examined using an inverted microscope (serial no. 321463; Leica). After being transfected with the si-NC or si-Kif4 for $48 \mathrm{~h}$, RAW264.7 cells were observed under light field at x200 magnification. Mouse insulin-like growth factor-1 (IGF-1) (100 ng/ml) was used to treat the cells for $36 \mathrm{~h}$ in si-NC and si-Kif4 groups, after 48-h transfection with si-NC or si-Kif4. IGF-1 was purchased from ProSpec-Tany TechnoGene, Ltd. (Ness Ziona, Israel).

Flow cytometric analysis. The RAW264.7 cells were collected and washed with cold phosphate-buffered saline (PBS). The cells were then incubated with monoclonal antibodies against CD11c (M10118-02B) and CD80 (M10801-09B) (both from Tianjin Sungene Biotech Co., Ltd., Tianjin, China) for $20 \mathrm{~min}$ in the dark. The cells were washed with cold PBS again and analyzed with a flow cytometer (FACSCalibur; Becton-Dickinson, Mountain View, CA, USA). A minimum of 10,000 events/sample were collected for analysis.

Reverse transcription-quantitative polymerase chain reaction ( $R T-q P C R)$ assays. Total RNA was extracted from the cells using an RNeasy Mini kit (Qiagen, Dusseldorf, Germany) in accordance with the RNeasy Mini Handbook. RNA was reverse transcribed using the ReverTra Ace qPCR RT kit (Toyobo Co., Ltd., Osaka, Japan). PCR analysis was performed with cDNA and the following primers: mouse VEGFR1 forward, 5'-CTTTCTCAAGTGCAGAGGGG-3' and reverse, 5'-TCATGTGCACAAGTTTGGGT-3'; mouse VEGF-A forward, 5'-GGGACCCCTTCGTCCTCTC-3' and reverse, 5'-GTCTCCTGGGGACAGAATTAGTG-3'; and mouse glyceraldehyde 3-phosphate dehydrogenase (GAPDH) forward, 5'-CCCACTAACATCAAATGGGG-3' and reverse, 5'-CCTTCCACAATGCCAAAGTT-3'.

The cDNA sample amplification was carried out using SYBR PremixEx Taq ${ }^{\mathrm{TM}}$ II (Tli RNaseH Plus; Takara, Dalian, China). The RT-PCR conditions were as follows: $3 \mathrm{sec}$ at $95^{\circ} \mathrm{C}$, followed by 40 cycles consisting of denaturing at $95^{\circ} \mathrm{C}$ for $5 \mathrm{sec}$, annealing at $60^{\circ} \mathrm{C}$ for $10 \mathrm{sec}$, and extending at $72^{\circ} \mathrm{C}$ for $15 \mathrm{sec}$. Relative quantification values were calculated using the $\Delta \Delta$ Cqmethod.

Western blot analysis. The cells were collected and lysed in ice-cold radioimmunoprecipitation assay (RIPA) lysis buffer containing $1 \mathrm{mM}$ phenylmethanesulfonyl fluoride (Solarbio, Beijing, China). The supernatant were collected following centrifugation at $10,000 \mathrm{rpm}$ for $15 \mathrm{~min}$ at $4^{\circ} \mathrm{C}$. The protein 
A

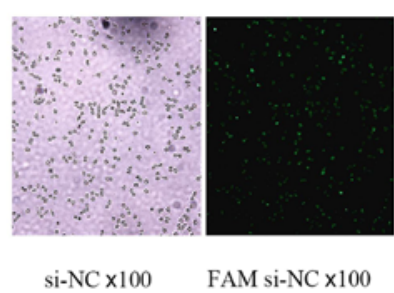

$\mathrm{D}$

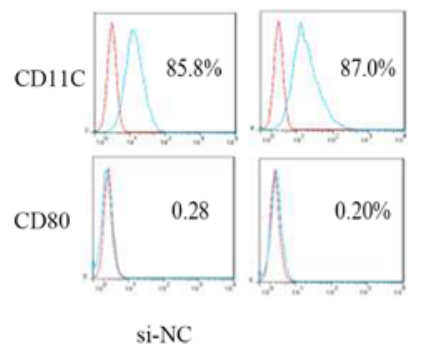

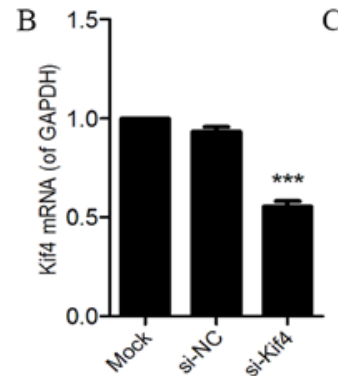

C

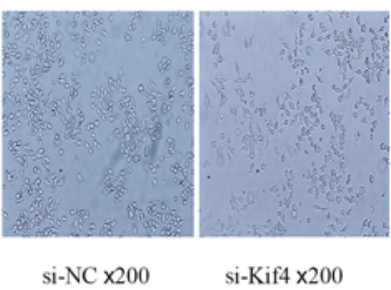

$\mathrm{F}$

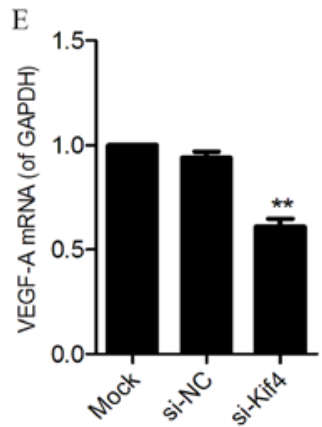

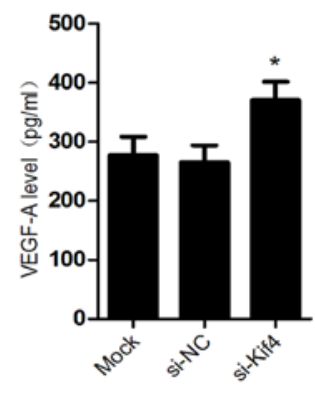

Figure 1. Effects of the silencing of kinesin superfamily protein 4 (Kif4) using siRNA (si-Kif4) on RAW264.7 cells. (A) RAW264.7 cells were transfected with green fluorescent FAM-labeled negative control siRNA (si-NC) for $6 \mathrm{~h}$, and were then observed under a light microscope (left panel) and fluorescence microscope (right panel). (B) The RAW264.7 cells were transfected with the Lipofectamine 2000 only (mock), si-NC or si-Kif4. After 24 h, the expression of Kif4 mRNA in the group transfected with si-Kif4 was decreased compared with the other two groups. (C) Cell morphology was examined under a microscope. (D) The levels of the costimulatory molecules, CD11c and CD80, are not obviously altered. (E) After 24 h, RT-PCR analysis was carried out to examine the vascular endothelial growth factor (VEGF) mRNA levels. (F) After 48 h, ELISA was carried out to determine the VEGF levels in the cell supernatant. Data are presented as the means \pm SD of 3 independent experiments. ${ }^{*} \mathrm{P}<0.05,{ }^{* *} \mathrm{P}<0.01,{ }^{* * * *} \mathrm{P}<0.001$ as compared with the control.

concentration was determined using a Micro $\mathrm{BCA}^{\mathrm{TM}}$ Protein Assay kit (Pierce, Rockford, IL, USA). Equal amounts of protein were loaded, separated by SDS-PAGE and transferred onto PVDF membranes (Invitrogen Life Technologies). For $2 \mathrm{~h}$, the membranes were blocked with $5 \%(\mathrm{w} / \mathrm{v})$ fat-free milk dissolved in Tris-buffered saline containing $0.05 \%$ Tween-20 (TBST). The membranes were then hybridized to a mouse anti-AKT antibody (1:1,000 \#9272), a phosphorylated Akt (p-Akt) antibody (1:1,000; \#9271) or GAPDH antibody (1:2,000; \#97166) (all from Cell Signaling Technology, Inc., Boston, MA, USA) at room temperature for $2 \mathrm{~h}$. The membranes were washed in TBST and then incubated with HRP-conjugated secondary antibody (1:2,000; ZDR-5307; ZSGB-BIO, Beijing, China). Blots were visualized using an ECL chemiluminescent kit (Millipore, Billerica, MA, USA). The bands were processed and analyzed using the FluorChem system (Alpha Innotech, Cell Biosciences Inc., Santa Clara, CA, USA).

Enzyme-linked immunosorbent assay (ELISA). The concentrations of mouse sVEGFR1 and VEGF-A in the cell culture medium were detected using ELISA kits (R\&D Systems, Inc., Minneapolis, MN, USA), according to the manufacturer's instructions. Briefly, $50 \mu \mathrm{l}$ assay diluent was added to each well and the same volume of serially diluted standards and samples were loaded on the plate followed by incubation for $2 \mathrm{~h}$ at room temperature on the shaker at $200 \mathrm{rpm}$. Excess antibodies were then removed 5 times with wash buffer, and incubated for $2 \mathrm{~h}$ with conjugate. The reaction was developed by substrate and the optical density was measured using a spectrophotometer (GeneQuant 100; Biochrom, Holliston, MA, America) at $450 \mathrm{~nm}$ (correcting at $540 \mathrm{~nm}$ ).
Statistical analysis. The values are expressed as the means \pm standard deviation (SD). Data were analyzed using the Student's t-test or by analysis of variance (ANOVA). A P-value $<0.05$ was considered to indicate a statistically significant difference.

\section{Results}

Silencing of Kif4 decreases VEGF-A mRNA expression, but increases VEGF-A levels in supernatant of RAW264.7 cells. The RAW264.7 cells were transfected with si-NC or FAM si-NC for $6 \mathrm{~h}$, and were observed under a fluorescence microscope. We observed green fluorescence in the RAW264.7 cells. This suggests that the FAM si-NC with green fluorescent tags was successfully transfected into the cells (Fig. 1A).

RNA interference targeting Kif4 was determined by RT-PCR. The reduction of Kif4 mRNA expression by $\sim 60 \%$ was observed in the si-Kif4 transfected cells compared to the si-NC- and Lipofectamine 2000 only-transfected cells (Fig. 1B). No morphological changes in the cells were observed at $48 \mathrm{~h}$ following transfection (Fig. 1C). In both the si-NC group and si-Kif4 group, RAW264.7 cell bodies were plump, oval and thin at the cell ends. Most of the cells exhibited two or fewer extended dendritic pseudopods. Flow cytometric analysis indicated that the expression of the costimulatory molecules, CD11c and CD80, was not obviously altered (Fig. 1D).

The results of RT-PCR revealed that at $24 \mathrm{~h}$ following transfection of the RAW264.7 cells with si-Kif4, VEGF-A mRNA expression was markedly decreased (Fig. 1E). However, at $48 \mathrm{~h}$ post-transfection, the level of VEGF-A was increased in the cell supernatant, as shown by ELISA (Fig. 1F), which was inconsistent with the decreased gene level. We hypothesized 

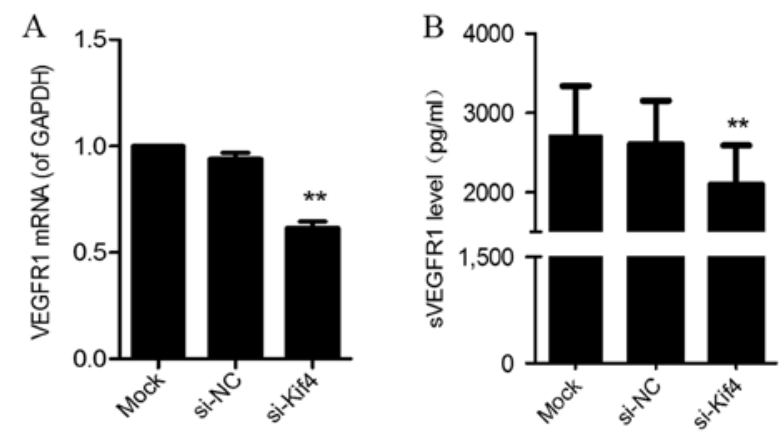

$\mathrm{C}$

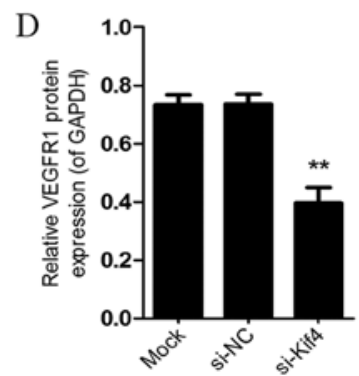

Figure 2. Expression of vascular endothelial growth factor (VEGF) receptor 1 (VEGFR1) and soluble form VEGFR1 (sVEGFR1) in RAW264.7 cells transfected with kinesin superfamily protein 4 (Kif4) siRNA (si-Kif4). (A) After $24 \mathrm{~h}, \mathrm{RT}-\mathrm{PCR}$ was carried out to measure the VEGFR1 mRNA levels in RAW264.7 cells. (B) After 48 h, RT-PCR was carried out to measure the VEGFR1 mRNA levels in RAW264.7 cells. (C) After $48 \mathrm{~h}$, western blot analysis was carried out to determine the VEGFR1 protein levels in RAW264.7 cells. (D) After $48 \mathrm{~h}$, ELISA was carried out to determine the levels of sVEGFR1 in different groups in the cell supernatant. Data are presented as the means \pm SD of 3 independent experiments. ${ }^{* *} \mathrm{P}<0.01$ as compared with control.

that this was due to a decrease in the level of sVEGFR1 in the cell supernatant, which is a decoy receptor of VEGF-A, which kept the levels of free VEGF-A from decreasing. Thus, we examined whether Kif4 regulates the levels of VEGFR1 and sVEGFR1 in the RAW264.7 cells.

Silencing of Kif4 using siRNA (si-Kif4) regulates the expression of sVEGFR1 and VEGFR1 in RAW264.7 cells. The results of RT-PCR revealed that at $24 \mathrm{~h}$ post-transfection, VEGFR1 mRNA expression was decreased (Fig. 2A). The results of ELISA also revealed that the level of sVEGFR1 was decreased in the cell supernatant following transfection with si-Kif4 (Fig. 2B). In addition, the results of western blot analysis indicated that the protein expression of VEGFR1 was decreased following transfection with si-Kif4 (Fig. 2C).

Silencing of Kif4 using siRNA (si-Kif4) regulates the levels of VEGFR1 and SVEGFR1 in RAW264.7 cells through the inhibition of the PI3K/Akt signaling pathway. We then explored the signaling mechanisms involved in the regulatory effects of si-Kif4 on the levels VEGFR1 and sVEGFR1 in RAW264.7 cells. We found that the levels of p-Akt significantly decreased at $48 \mathrm{~h}$ post-transfection in the si-Kif4-transfected group compared with the mock- and si-NC-transfected groups (Fig. 3A). IGF-1 is the specific agonist of PI3K/Akt. Thus, we also used IGF-1 to examine its effects on the transfected cells. The results revealed a high level of p-Akt at $30 \mathrm{~min}$ after the addition of IGF-1 $(100 \mathrm{ng} / \mathrm{ml})$ in the si-Kif4-transfected group; the level remained high at $60 \mathrm{~min}$ (Fig. 3B).
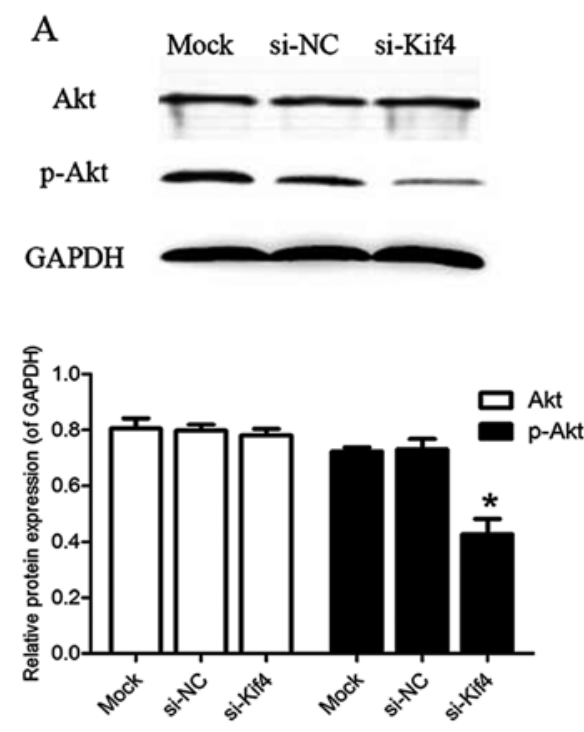

B
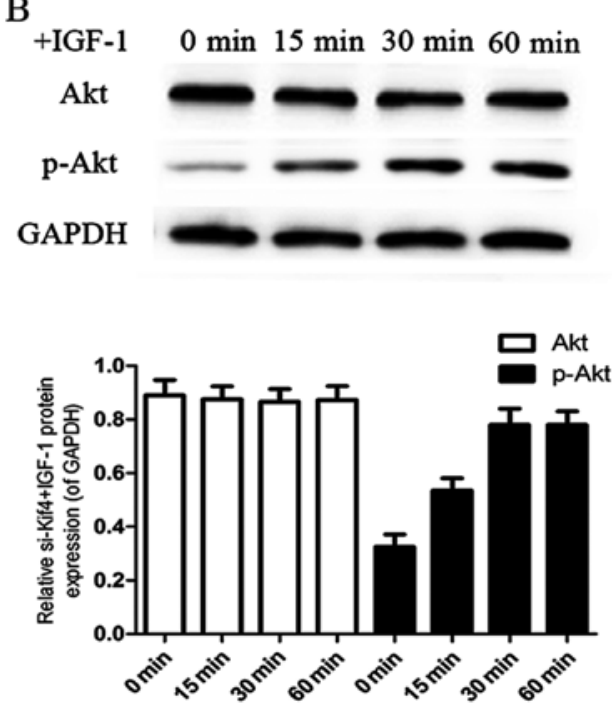

Figure 3. Effects of the silencing of kinesin superfamily protein 4 (Kif4) with siRNA (si-Kif4) on the PI3K/Akt signaling in RAW264.7 cells. (A) After $48 \mathrm{~h}$, western blot analysis was used to detect the levels of Akt and phosphorylated Akt (p-Akt). (B) Western blot analysis of a time course of Akt and p-Akt in the si-Kif4-transfected group also treated with insulin-like growth factor-1 (IGF-1) (100 ng/ml) and with GAPDH as the loading control. Data are presented as the means \pm SD of 3 independent experiments. ${ }^{*} \mathrm{P}<0.05$ as compared with the control.

The suppressive effects of the silencing of Kif4 on VEGFRI $m R N A$ expression and the level of SVEGFRl are abrogated by $I G F-1$. Following the addition of IGF-1 (100 ng/ml) for $36 \mathrm{~h}$ in the si-NC- and si-Kif4-transfected groups, we detected a significant increase in the VEGFR1 mRNA (Fig. 4A), and VEGFR1 protein levels (Fig. 4B); the levels of sVEGFR1 also increased in the cell supernatant in the si-Kif4-transfected group (Fig. 4C).

The addition of IGF-1 alters the mRNA expression of VEGF in si-Kif4-transfected cells, as well as the level of VEGF-A in the cell supernatant. Following the addition of IGF-1 (100 ng/ $\mathrm{ml}$ ) for $36 \mathrm{~h}$, we found that VEGF-A mRNA expression was did significantly altered between the si-NC- and si-Kif4-trans- 

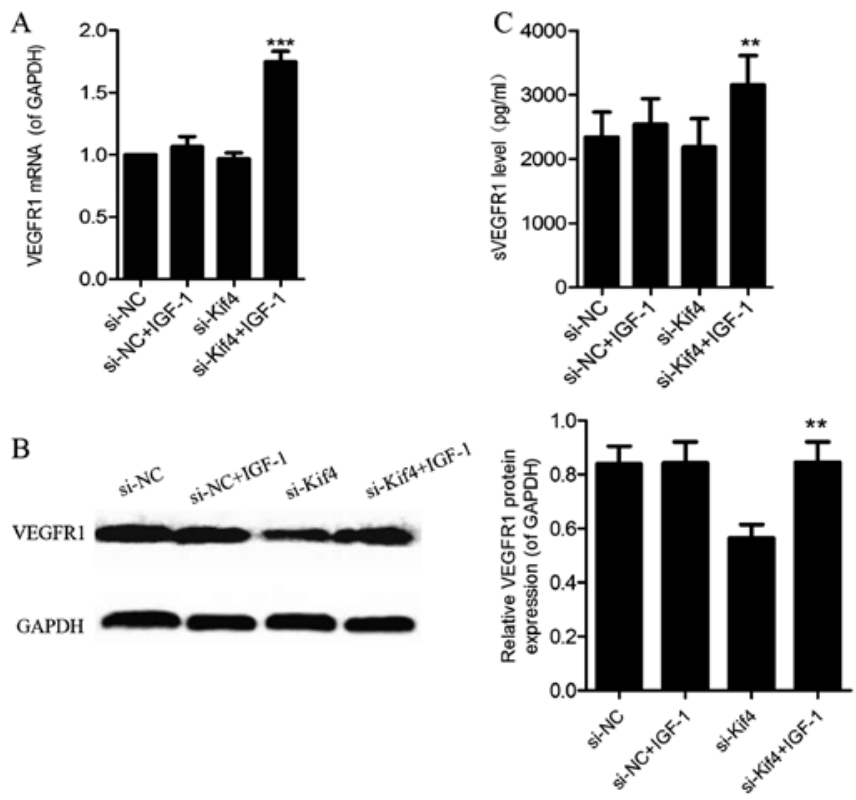

Figure 4. The use of insulin-like growth factor-1 (IGF-1) abrogates the suppressive effects of the silencing of kinesin superfamily protein 4 (Kif4) on vascular endothelial growth factor (VEGF) receptor 1 (VEGFR1) mRNA and soluble form VEGFR1 (sVEGFR1). IGF-1 (100 ng/ml) was used to treat the cells for $36 \mathrm{~h}$ in the negative control siRNA (si-NC)- and Kif4 siRNA (siKif4)-transfected groups. (A) RT-PCR analysis of VEGFR1 mRNA levels in RAW264.7 cells. (B) Western blot analysis of VEGFR1 protein levels in RAW264.7 cells. (C) ELISA of the levels of sVEGFR1 in the cell supernatant. Data are presented as the means $\pm \mathrm{SD}$ of 3 independent experiments. ${ }^{* *} \mathrm{P}<0.01$, ${ }^{* * *} \mathrm{P}<0.001$ as compared with the control.
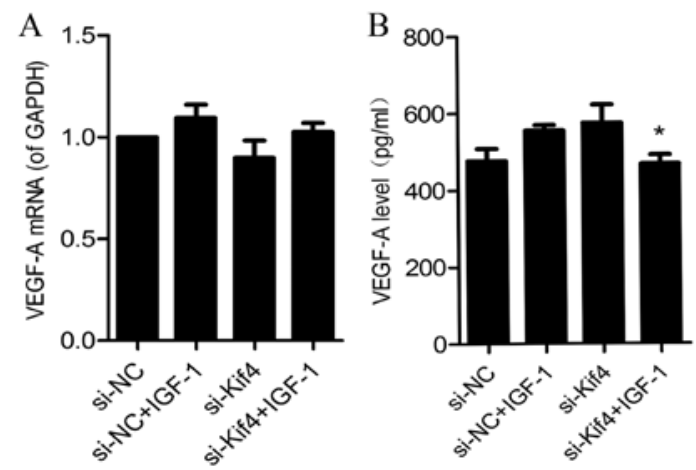

Figure 5. Expression of vascular endothelial growth factor-A (VEGF-A) in different groups. Insulin-like growth factor-1 (IGF-1) $(100 \mathrm{ng} / \mathrm{ml})$ was added for $36 \mathrm{~h}$. (A) RT-PCR analysis of VEGF-A mRNA levels in RAW264.7 cells. (B) ELISA of the levels of VEGF-A in the cell supernatant. Data are presented as the means $\pm \mathrm{SD}$ of 3 independent experiments. ${ }^{*} \mathrm{P}<0.05$ as compared with the control.

fected groups (Fig. 5A); however, the expression of VEGF-A in the cell supernatant was decreased in the si-Kif4-transfected group (Fig. 5B).

\section{Discussion}

Kinesin superfamily motor proteins can not only directionally transport various cargos, but also play significant roles in various processes fundamental for life, such as angiogenesis (1). KIF13B has been shown to be an essential molecular motor to the trafficking of VEGFR2 from the Golgi apparatus to the endothelial cell surface to mediate angiogenesis (27).
Kif11/Eg5 plays a role in endothelial cell adhesion, migration and angiogenesis (28).

In this study, our results demonstrated that the silencing of Kif4 regulated the expression of VEGFR1, sVEGFR1 and VEGF, and inhibited the activation of the PI3K/Akt signaling pathway in RAW264.7 cells, which may be one of the mechanisms responsible for Kif4 participating in the regulation of angiogenesis.

Initially, we found that the mRNA expression of VEGF-A was decreased in the RAW264.7 cells (Fig. 1E), but the protein level of VEGF-A was significantly higher in the cell supernatant in the cells transfected with si-Kif4. To explain these seemingly contradictory results, we considered whether this occurred due to the fact that the level of sVRGFR1, a decoy receptor of VEGF-A, was decreased in the cell supernatant. The silencing of Kif4 decreased the VEGFR1 mRNA, sVEGFR1 and VEGFR1 protein expression (Fig. 2A-C). By contrast, the transmembrane form of VEGFR1 was significantly increased (data not shown). This indicated that the silencing of Kif4 not only modulated the protein expression of VEGFR1 in RAW264.7 cells, but also regulated the proportion of the transmembrane form of VEGFR1 and secreted forms. However, the mechanisms involved require further investigation.

The PI3K/Akt pathway is a cell survival pathway, which regulates gene expression and cell metabolism, as well as cytoskeletal rearrangements. The PI3K/Akt pathway has been implicated in various human diseases, including diabetes and cancer $(29,30)$.

In the human placental hypoxia model, the PI3K/Akt pathway has been shown to be significantly involved in the regulation and expression sVEGFR1 (31). Granulocyte-macrophage colony-stimulating factor (GM-CSF) can stimulate human monocytes to produce sVEGFR1, inhibiting the biological effects of VEGF-A, by activating the PI3K/Akt pathway (32). Human monocyte $\mathrm{Fc} \gamma$ receptor activation can also promote the secretion of sVEGFR1 via the PI3K/Akt pathway, thereby inhibiting human umbilical vein endothelial cell (HUVEC) tube formation effect (33).

In order to understand the molecular mechanisms responsible for the regulation of VEGFR1 and sVEGFR1 in RAW264.7 cells by the silencing of Kif4, we investigated PI3K/Akt signaling. Transfection with si-Kif4 inhibited the PI3K/Akt signaling pathway at $24 \mathrm{~h}$ (data not shown), and the inhibitory effect was more obvious at $48 \mathrm{~h}$ (Fig. 3A). IGF-1 is the specific agonist of PI3K/Akt (34) (Fig. 3B). The addition of IGF-1 increased the expression of VEGFR1 mRNA, VEGFR1 protein andthe level of sVEGFR1 in the si-Kif4-transfected cells and in the cell supernatant (Fig. 4).

We also found that following the activation of the PI3K/ Akt signaling pathway, the level of VEGF-A mRNA was not significantly altered (Fig. 5A); however, the level of VEGF-A was significantly decreased in the cell supernatant (Fig. 5B). We considered that this was due to the increased level of sVEGFR1 which combined with VEGF-A, so that free VEGF-A was reduced.

In conclusion, in this study, to the best of our knowledge, we demonstrated for the first time that the silencing of Kif4 regulated VRGFR1 mRNA transcription and sVEGFR1 production in monocytes via the PI3K/Akt signaling pathway, which may be one of the mechanisms responsible for these processes. Our 
results not only provide a deeper understanding of the function of Kif4, but also enhance our understanding of VEGFR1 and sVEGFR1 generation mechanisms in monocytes. These data may help to explain the role of monocytes/macrophages in angiogenesis and provide valuable information on treatment strategies for diseases, such as cancer and inflammation.

\section{Acknowledgements}

The present study was supported by the Natural Science Foundation of China (grant nos. 81271105, 31470885 and 31270971)

\section{References}

1. Hirokawa N, Noda Y, Tanaka Y and Niwa S: Kinesin superfamily motor proteins and intracellular transport. Nat Rev Mol Cell Biol 10: 682-696, 2009.

2. Lawrence CJ, Dawe RK, Christie KR, Cleveland DW, Dawson SC, Endow SA, Goldstein LS, Goodson HV, Hirokawa N, Howard J, et al: A standardized kinesin nomenclature. J Cell Biol 167: 19-22, 2004.

3. Sekine Y, Okada Y, Noda Y,Kondo S, Aizawa H, Takemura R and Hirokawa N: A novel microtubule-based motor protein (KIF4) for organelle transports, whose expression is regulated developmentally. J Cell Biol 127: 187-201, 1994.

4. Kurasawa Y, Earnshaw WC, Mochizuki Y, Dohmae N and Todokoro K: Essential roles of KIF4 and its binding partner PRC1 in organized central spindle midzone formation. EMBO J 23: 3237-3248, 2004.

5. Zhu C and Jiang W: Cell cycle-dependent translocation of PRC1 on the spindle by Kif4 is essential for midzone formation and cytokinesis. Proc Natl Acad Sci USA 102: 343-348, 2005.

6. Wu G, Zhou L, Khidr L, Guo XE, Kim W, Lee YM, Krasieva T and Chen PL: A novel role of the chromokinesin Kif4A in DNA damage response. Cell Cycle 7: 2013-2020, 2008.

7. Martinez NW, Xue X, Berro RG, Kreitzer G and Resh MD: Kinesin KIF4 regulates intracellular trafficking and stability of the human immunodeficiency virus type $1 \mathrm{Gag}$ polyprotein. J Virol 82: 9937-9950, 2008.

8. Bernasconi P, Cappelletti C, Navone F, Nessi V, Baggi F, Vernos I, Romaggi S, Confalonieri P, Mora M, Morandi L, et al: The kinesin superfamily motor protein KIF4 is associated with immune cell activation in idiopathic inflammatory myopathies. $\mathbf{J}$ Neuropathol Exp Neurol 67: 624-632, 2008.

9. Midorikawa R, Takei Y and Hirokawa N: KIF4 motor regulates activity-dependent neuronal survival by suppressing PARP-1 enzymatic activity. Cell 125: 371-383, 2006.

10. Mazumdar M, Lee JH, Sengupta K, Ried T, Rane S and Misteli T: Tumor formation via loss of a molecular motor protein. Curr Biol 16: 1559-1564, 2006.

11. Carmeliet P: Angiogenesis in life, disease and medicine. Nature 438: 932-936, 2005.

12. Olsson AK, Dimberg A, Kreuger J and Claesson-Welsh L: VEGF receptor signalling - in control of vascular function. Nat Rev Mol Cell Biol 7: 359-371, 2006.

13. Cao Y: Positive and negative modulation of angiogenesis by VEGFR1 ligands. Sci Signal 2: re1, 2009.

14. Ahmad S and Ahmed A: Elevated placental soluble vascular endothelial growth factor receptor-1 inhibits angiogenesis in preeclampsia. Circ Res 95: 884-891, 2004.

15. Wu FT, Stefanini MO, Mac Gabhann F, Kontos CD, Annex BH and Popel AS: A systems biology perspective on sVEGFR1: its biological function, pathogenic role and therapeutic use. J Cell Mol Med 14: 528-552, 2010.

16. Geissmann F, Manz MG, Jung S, Sieweke MH, Merad M and Ley K: Development of monocytes, macrophages, and dendritic cells. Science 327: 656-661, 2010.
17. Clauss M, Weich H, Breier G, Knies U, Röckl W, Waltenberger J and Risau W: The vascular endothelial growth factor receptor Flt-1 mediates biological activities. Implications for a functional role of placenta growth factor in monocyte activation and chemotaxis. J Biol Chem 271: 17629-17634, 1996.

18. Sawano A, Iwai S, Sakurai Y, Ito M, Shitara K, Nakahata T and Shibuya M: Flt-1, vascular endothelial growth factor receptor 1 , is a novel cell surface marker for the lineage of monocyte-macrophages in humans. Blood 97: 785-791, 2001.

19. Crowther M, Brown NJ, Bishop ET and Lewis CE: Microenvironmental influence on macrophage regulation of angiogenesis in wounds and malignant tumors. J Leukoc Biol 70: 478-490, 2001.

20. Hiratsuka S, Minowa O, Kuno J, Noda T and Shibuya M: Flt-1 lacking the tyrosine kinase domain is sufficient for normal development and angiogenesis in mice. Proc Natl Acad Sci USA 95: 9349-9354, 1998.

21. Lewis CE and Pollard JW: Distinct role of macrophages in different tumor microenvironments. Cancer Res 66: 605-612, 2006.

22. Kerber M, Reiss Y, Wickersheim A, Jugold M, Kiessling F, Heil M, Tchaikovski V, Waltenberger J, Shibuya M, Plate KH, et al: Flt-1 signaling in macrophages promotes glioma growth in vivo. Cancer Res 68: 7342-7351, 2008.

23. Peppicelli S, Bianchini F and Calorini L: Inflammatory cytokines induce vascular endothelial growth factor-C expression in melanoma-associated macrophages and stimulate melanoma lymph node metastasis. Oncol Lett 8: 1133-1138, 2014.

24. Murakami M, Iwai S, Hiratsuka S, Yamauchi M, Nakamura K, Iwakura Y and Shibuya M: Signaling of vascular endothelial growth factor receptor-1 tyrosine kinase promotes rheumatoid arthritis through activation of monocytes/macrophages. Blood 108: 1849-1856, 2006.

25. Murakami M, Zheng Y, Hirashima M, Suda T, Morita Y, Ooehara J, Ema H, Fong GH and Shibuya M: VEGFR1 tyrosine kinase signaling promotes lymphangiogenesis as well as angiogenesis indirectly via macrophage recruitment. Arterioscler Thromb Vasc Biol 28: 658-664, 2008.

26. Matsui M, Takeda Y, Uemura S, Matsumoto T, Seno A, Onoue K, Tsushima H, Morimoto K, Soeda T, Okayama S, et al: Suppressed soluble Fms-like tyrosine kinase-1 production aggravates atherosclerosis in chronic kidney disease. Kidney Int 85: 393-403, 2014.

27. Yamada KH, Nakajima Y, Geyer M, Wary KK, Ushio-Fukai M, Komarova Y and Malik AB: KIF13B regulates angiogenesis through Golgi to plasma membrane trafficking of VEGFR2. J Cell Sci 127: 4518-4530, 2014.

28. Exertier P, Javerzat S, Wang B, Franco M, Herbert J, Platonova N, Winandy M, Pujol N, Nivelles O, Ormenese S, et al: Impaired angiogenesis and tumor development by inhibition of the mitotic kinesin Eg5. Oncotarget 4: 2302-2316, 2013.

29. Cantley LC: The phosphoinositide 3-kinase pathway. Science 296: 1655-1657, 2002.

30. Hemmings BA and Restuccia DF: The PI3K-PKB/Akt pathway. Cold Spring Harb Perspect Biol 7: 1-3, 2015.

31. Park JK, Jeong JW, Kang MY, Baek JC, Shin JK, Lee SA, Choi WS, Lee JH and Paik WY: Inhibition of the PI3K-Akt pathway suppresses sFlt1 expression in human placental hypoxia models in vitro. Placenta 31: 621-629, 2010.

32. Eubank TD, Roberts R, Galloway M, Wang Y, Cohn DE and Marsh CB: GM-CSF induces expression of soluble VEGF receptor-1 from human monocytes and inhibits angiogenesis in mice. Immunity 21: 831-842, 2004.

33. Justiniano SE, Elavazhagan S, Fatehchand K, Shah P, Mehta P, Roda JM, Mo X, Cheney C, Hertlein E, Eubank TD, et al: Fc $\gamma$ receptor-induced soluble vascular endothelial growth factor receptor-1 (VEGFR-1) production inhibits angiogenesis and enhances efficacy of anti-tumor antibodies. J Biol Chem 288: 26800-26809, 2013.

34. Michell BJ, Griffiths JE, Mitchelhill KI, Rodriguez-Crespo I, Tiganis T, Bozinovski S, de Montellano PR, Kemp BE and Pearson RB: The Akt kinase signals directly to endothelial nitric oxide synthase. Curr Biol 9: 845-848, 1999. 